\title{
Sporadic hyperekplexia due to self-limiting brainstem encephalopathy
}

This article was published in the following Dove Press journal:

Neuropsychiatric Disease and Treatment

9 October 2017

Number of times this article has been viewed

\author{
Dilek Yilmaz' \\ Bülent Cengiz ${ }^{2}$ \\ 'Department of Neurology, Konya \\ Numune State Hospital, Konya, \\ ${ }^{2}$ Department of Neurology, Gazi \\ University Faculty of Medicine, \\ Ankara, Turkey
}

\begin{abstract}
Hyperekplexia is a rare movement disorder characterized by pathologically exaggerated response to unexpected stimuli. It is differentiated from the normal startle reflex by its lower threshold, higher intensity, and resistance to habituation. Many of the acquired hyperekplexias result from brainstem involvement such as encephalitis, infarct, hemorrhage, pontocerebellar hypoplasia and medullary compression. This case report depicts a rare manifestation of hyperekplexia. The unusual aspect of this case was the vocalization that was reproduced in response to startling stimuli. Startle induced vocalization is not a part of the classical hyperekplexia description. When faced with a patient with pathologically exaggerated response to unexpected stimuli, the physician should consider this rare condition in the differential diagnosis, and also keep in mind that the disease may present with features different from those listed in the textbooks.
\end{abstract}

Keywords: hyperekplexia, startle, brainstem encephalopathy

\section{Introduction}

Hyperekplexia is a rare movement disorder characterized by pathologically exaggerated response to unexpected stimuli, and it is differentiated from the normal startle reflex by its lower threshold, higher intensity, and resistance to habituation. ${ }^{1}$ Hyperekplexia can result from either hereditary or acquired causes. Many of the acquired hyperekplexias are symptomatic and result from brainstem involvement such as encephalitis, infarct, hemorrhage, pontocerebellar hypoplasia, and medullary compression. ${ }^{2}$

Our aim was to draw attention to this rarely encountered condition through this case report of a 48-year-old patient with sporadic hyperekplexia who initially presented with brainstem involvement and who was diagnosed with the help of a series of electrodiagnostic tests.

\section{Case report}

A 48-year-old male admitted to the emergency department with unbalanced gait followed by diplopia, difficulty speaking, and swallowing. He had admitted to the ear, nose, and throat (ENT) outpatient clinic 5 days ago, where he was diagnosed with left peripheral facial palsy and was started on oral steroids. His family history was unremarkable. Neurological examination revealed dysarthria, restriction of left eye abduction, weakness of the masseter muscle, left peripheral facial palsy, tongue weakness on the left side, and ataxia. Because the neurological examination of the patient was suggestive of brainstem involvement, the differential diagnosis included paraneoplastic and viral encephalitis, congenital posterior fossa malformation, brainstem infarct or hemorrhage, vascular compression, intracranial mass lesion, sarcoidosis, and multiple sclerosis. Complete blood count, liver and kidney function tests, and thyroid function tests were
Correspondence: Dilek Yilmaz Department of Neurology, Konya Numune State Hospital, 42060, Selçuklu, Konya, Turkey

Email dilekyillmaz@gmail.com 
all within normal physiological limits. Written informed consent has been provided by the patient to have the case details and any accompanying images and/or video published. The images were blurred to render the patient anonymous. The patient showed generalized involuntary brief jerks affecting the whole body. Segment 1, tactile stimulation of forehead results in generalized startle response. Segment 2, tactile stimulation of foot results in only restricted flexion on the legs. Segment 3, sudden, unexpected sound provokes patient's startle reaction. Segment 4, neurologic examination 3 months after discharge.

Paraneoplastic antibodies in serum and cerebrospinal fluid (CSF) were negative. CSF examination was normal except positive oligoclonal bands, which were detected by isoelectric focusing and immunoblotting. All cultures and serologies were negative. There was no evidence of vasculitis or rheumatologic disease. The serum angiotensin-converting enzyme (ACE) level was $34.00 \mathrm{U} / \mathrm{L}$ (8-52.00). Serum and urine immunoelectrophoresis results were within normal physiological limits. Magnetic resonance imaging (MRI) of the brain, cervical, and thoracic spinal cord was normal with no enhancement after gadolinium. Electroencephalogram (EEG) was within normal physiological limits. The patient was started on low molecular weight heparin and symptomatic treatment.

One month after admission, the abnormal findings on the initial neurologic examination improved, except the restriction of left eye abduction. However, the patient developed brisk patella and Achilles reflexes, lower extremity spasticity, bilateral Babinski's sign, Achilles clonus, and trismus. He also manifested generalized involuntary brief jerks affecting the whole body. Jerks were triggered by sudden unexpected noises or by touching the perioral region and forehead, and continued during sleep, causing him to wake. During these attacks, blinking, flexion of neck, trunk, elbows, and knees, shoulder abduction, and involuntary voice spasms have been observed (Video S1). The patient was investigated for possible infectious and malignant etiologies, and the results were negative. Routine fluorine-18-2-fluoro-2-deoxy-D-glucose positron emission tomography/computed tomography (18 FDG PET/CT) was within normal limits. Persistent abnormal startle response was detected in response to repeated unexpected acoustic stimuli. Simultaneous electromyographic (EMG) recordings showed an early contraction in sternocleidomastoid muscle, followed by slow recruitment of limb muscles in a rostrocaudal order (Figure 1A). Moreover, the conduction time was prolonged for biceps brachii and tibialis anterior muscles.

There was no habituation on reflex motor responses to repeated stimulation (Figure $1 \mathrm{~B}$ ). EEG, posterior tibial somatosensory-evoked potential (SEP), and blink reflexes were all normal. EMG of the right tibialis anterior, first dorsal interosseous, T9-10 paraspinal muscles, and nerve conduction studies of the median, ulnar, sural, peroneal, and posterior tibial nerves were all normal.

The patient was treated with clonazepam ( $2 \mathrm{mg} /$ day $)$ and carbamazepine $(600 \mathrm{mg} /$ day $)$ orally. Over the next month, he improved rapidly and his drug doses were gradually decreased. Three months after discharge, the patient was asymptomatic and his medication was tapered off over a 4-week period (Video S1).

\section{Discussion}

Hyperekplexia is characterized by an accentuated response to unexpected tactile or auditory stimuli. Sporadic hyperekplexia is a rare condition which may be the result of brainstem or diffuse cerebral pathologies.

The clinical and electrophysiological findings of our patient indicate brainstem involvement. Following multiple cranial nerve palsy, the patient developed pyramidal signs in the lower limb, trismus, and generalized involuntary brief jerks affecting the whole body. The results of the neurophysiological studies showed that conduction in the spinal cord was slower than conduction in the brainstem. There was also prolongation of conduction time between arm and leg muscles. These are typical findings in hyperekplexia. ${ }^{1}$ The great extent and resistance to habituation of jerks serve to distinguish our patient's involuntary movements from normal startle reflex. Brainstem reticular reflex myoclonus is another movement disorder characterized by generalized axial myoclonic jerks. Although this disorder manifests with brief jerks, some features of our patient are incompatible with brainstem reticular reflex myoclonus. First, there were not spontaneous jerks in our patient, which are common for brainstem reticular reflex myoclonus. Second, in our patient, jerks were induced by brief taps on the perioral region and forehead. By contrast, jerks are generally elicited by taps on the distal limbs in the brainstem reticular reflex myoclonus. ${ }^{2}$ Third, slow efferent spinal conduction in our patient can be differentiated from the brainstem reticular reflex myoclonus in which efferent spinal conduction is rapid. ${ }^{1}$ Distinguishing features of our patient from stiff-person syndrome are the absence of spasms on axial muscles and involvement of face during jerks.

Many of the acquired hyperekplexias result from brainstem involvement such as encephalitis, infarct, hemorrhage, pontocerebellar hypoplasia, medullary compression, sarcoidosis, and multiple sclerosis. ${ }^{2}$ We could not demonstrate any vascular or structural abnormality on MRI and magnetic 
A

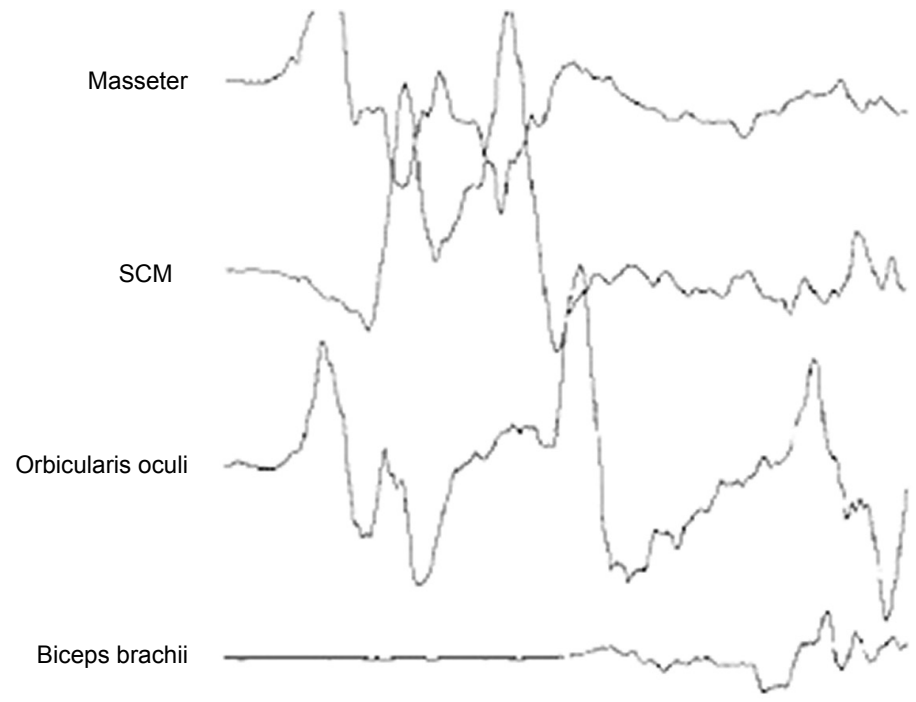

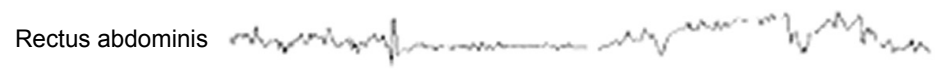

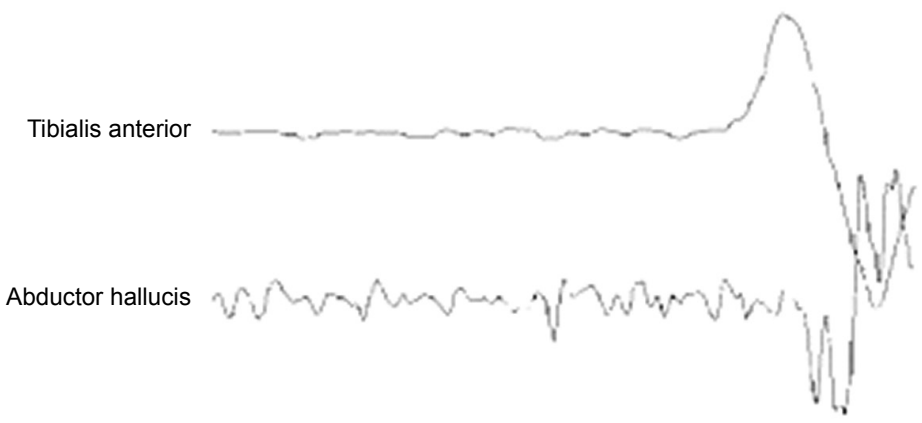

$0.5 \mathrm{mV}$

$10 \mathrm{~ms}$

B

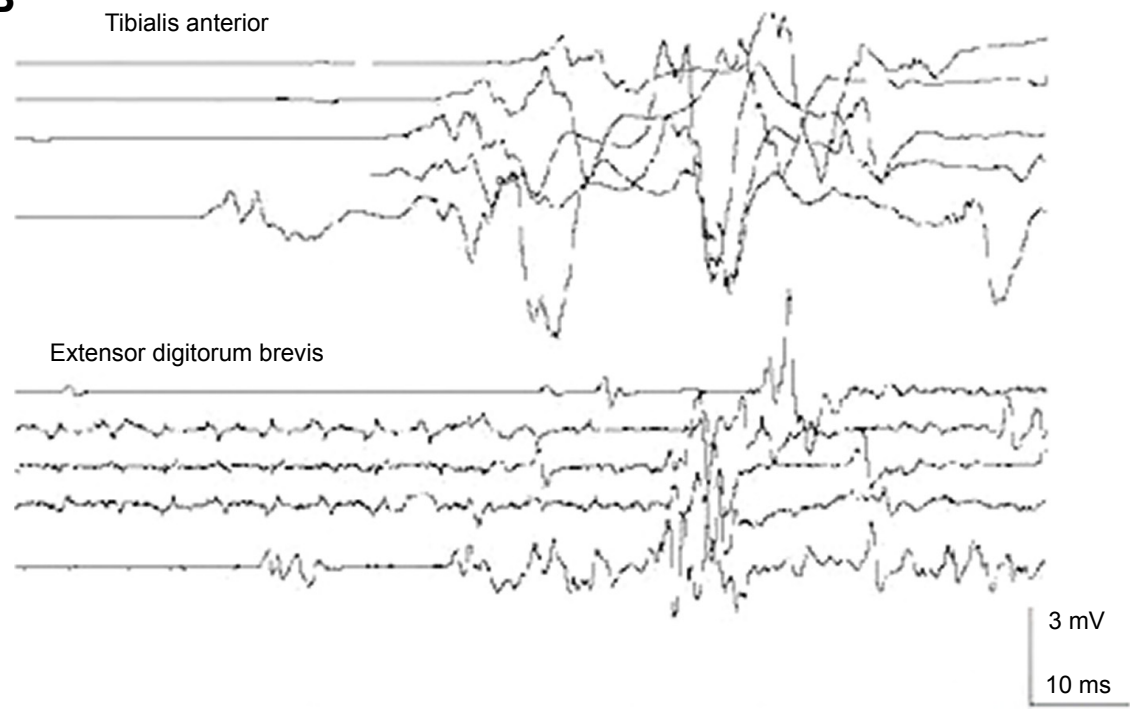

Figure I (A) EMG activity in the abnormal startle response evoked by unexpected acoustic stimulation. Each trial was started at the point of acoustic stimulation. EMG activity was first seen in the SCM muscle and followed by orbicularis oculi, masseter, biceps brachii, rectus abdominis, tibialis anterior, and abductor hallucis muscles. All traces reflect single trial. (B) EMG records of the startle response elicited by unexpected acoustic stimulation. Responses are not habituated after repeated stimulations.

Abbreviations: EMG, electromyographic; SCM, sternocleidomastoid. 
resonance angiography (MRA) of the patient. Multiple sclerosis and neurosarcoidosis were ruled out because the clinical signs and symptoms, the absence of MRI abnormality, and spontaneous recovery without immune-mediated treatment were all inconsistent with these diseases. Positive oligoclonal band seen in our patient may be due to an inflammatory process of brainstem involvement.

The unusual aspect of this case was the vocalization that was reproduced in response to startling stimuli. Startleinduced vocalization is not a part of the classical hyperekplexia description. Since the jerks were not painful, it is not reasonable to conclude that these vocalizations are related to pain or pain-induced anxiety. A possible explanation for this unusual finding may be culture-specific startle syndromes such as "Latah" in Indonesia and Malaysia and the "Jumping Frenchmen of Maine," which are characterized by non-habituating exaggerated startle responses triggered by, for instance, sound. Following being startled, there may be various behavioral responses, including "forced obedience," echolalia, echopraxia, and coprolalia. ${ }^{3}$ The absence of these epiphenomena in our patient and the complete resolution of the jerks with symptomatic treatment are inconsistent features with these syndromes.

Although clinical neurological signs and neurophysiological studies indicated a brainstem pathology, extensive investigation failed to reveal the underlying pathology. In the literature, there are two reports of brainstem encephalopathy with undefined etiology, manifesting with trismus and hyperekplexia. ${ }^{4,5}$ Like our patient, both of these two patients demonstrated hyperekplexia within 1-3 months after brainstem involvement. Trismus is another common finding in these two patients and our patient. However, in contrast to these two patients, hyperekplexia was completely resolved in our patient after 3 months of treatment with clonazepam.

\section{Conclusion}

The case presented in this study depicts a rare manifestation of hyperekplexia with brainstem involvement, possibly of inflammatory origin. The case was treated effectively with clonazepam.

\section{Disclosure}

The authors report no conflicts of interest in this work.

\section{References}

1. Brown P, Rothwell JC, Thompson PD, Britton TC, Day BL, Marsden CD. The hyperekplexias and their relationship to the normal startle reflex. Brain. 1991;114(pt 4):1903-1928.

2. Bakker MJ, van Dijk JG, van den Maagdenberg AM, Tijssen MA. Startle syndromes. Lancet Neurol. 2006;5(6):513-524.

3. Dreissen YE, Tijssen MA. The startle syndromes: physiology and treatment. Epilepsia. 2012;53(suppl 7):3-11.

4. Kellett MW, Humphrey PRD, Tedman BM, Steiger MJ. Hyperekplexia and trismus due to brainstem encephalopathy. J Neurol Neurosurg Psychiatry. 1998;65(1):122-125.

5. van de Warrenburg BP, Cordivari C, Brown P, Bhatia KP. Persisting hyperekplexia after idiopathic, self-limiting brainstem encephalopathy. Mov Disord. 2007;22(7):1017-1020.
Neuropsychiatric Disease and Treatment

\section{Publish your work in this journal}

Neuropsychiatric Disease and Treatment is an international, peerreviewed journal of clinical therapeutics and pharmacology focusing on concise rapid reporting of clinical or pre-clinical studies on a range of neuropsychiatric and neurological disorders. This journal is indexed on PubMed Central, the 'PsycINFO' database and CAS,

\section{Dovepress}

and is the official journal of The International Neuropsychiatric Association (INA). The manuscript management system is completely online and includes a very quick and fair peer-review system, which is all easy to use. Visit http://www.dovepress.com/testimonials.php to read real quotes from published authors. 